\title{
LAS COMPETENCIAS DOCENTES. UN ESTUDIO DE CASO REALIZADO EN LA FACULTAD DE CIENCIAS SOCIALES DE LA UNA
}

\section{THE TEACHING COMPETENCIES. A CASE STUDY DONE IN THE SOCIAL SCIENCE FACULTY AT UNA}

\author{
Carolina España Chavarríal \\ cespanac@yahoo.com.mx
}

\begin{abstract}
Resumen
Este artículo se ha elaborado a partir de los resultados obtenidos en la investigación doctoral realizada sobre "Las competencias explícitas y subyacentes en las prácticas docentes promovidas en la Facultad de Ciencias Sociales de la Universidad Nacional de Costa Rica”. La intención es que este estudio responda al aporte sobre las posibilidades y retos del tan esperado cambio educativo. Para ello se toma en cuenta el contexto social y sus demandas a la educación con el fin de dar las respuestas competentes a los desafíos que se le imponen. La figura protagónica del estudio es el cuerpo docente, como elemento clave para darle sentido al aprendizaje promovido a partir del desarrollo, la internalización y puesta en práctica de competencias renovadas, las cuales le permitan analizar, sintetizar, razonar críticamente, fundamentar y relacionar su pensamiento y el del colectivo estudiantil al que se debe. En la investigación se desarrolló un "campo o espacio común" donde intervenían de manera confluyente varias líneas de estudio: a. Las competencias esenciales, con el estudio de autores y el ejercicio pedagógico del planteamiento teórico/práctico de los docentes; $b$. El discurso de los estudiantes sobre el significado de las competencias para su aprendizaje y su desarrollo profesional y c. El perfil curricular por competencias tomado en la documentación institucional; obteniendo como uno de los resultados más importantes, la necesidad de vislumbrar la coherencia entre las competencias explícitas promovidas por el grupo docente y la puesta en escena de estas competencias en el proceso de construcción autónoma del conocimiento.

Palabras clave: Competencias docentes, Sociedad del conocimiento, Aprendizaje, Autonomía profesional, Desarrollo profesional, Formación docente, Educación superior, Construcción del conocimiento, Práctica reflexiva.
\end{abstract}

\begin{abstract}
This article has been elaborated based on the results obtained from the doctoral research on "The underlying and explicit competence in teaching practices promoted in the Social Science Faculty at the Universidad Nacional of Costa Rica". The intention is that this study responds to the contribution on the possibilities and challenges of the much-anticipated educational changes. To cope with the research objectives the social context and its educational demands were taken into account in order to give competent answers to the challenges imposed. The teaching staff was the target of the study, as the key element that gives meaning to the learning promoted derived from the development, internalization and implementation of renewed competencies, which allow them to analyze, synthesize, reason critically, explain and relate their thinking and the student's one. In the research a "field or common space" where several lines of study took place: a. Essential competencies, with the study of
\end{abstract}

1 Académica e investigadora de la Facultad de Ciencias Sociales de la Universidad Nacional. 
authors and the pedagogical approach derived from the theoretical/practical exercise done by teachers; $b$. The students' significance about the meaning and importance of the competencies for their learning and professional development, and c. Competency-based curriculum profile taken in the institutional documentation; obtaining one of the most important results, and the need for consistency between the explicit competencies promoted by the educational group and those display in the process of autonomous knowledge construction.

Key words: Teaching skills, Knowledge society, Learning, Professional autonomy, Professional development, Teacher training, Higher education, Building knowledge, Reflexive practice.

\section{Introducción}

Los retos que la sociedad del conocimiento le impone a la educación, implican una trasformación conceptual y procedimental de los contextos de aprendizaje, en este caso específico, en la Universidad Nacional de Costa Rica (UNA), de la Facultad de Ciencias Sociales (FCS), centro donde fue realizada la presente investigación doctoral. En este sentido se escogió a la Universidad Nacional como el universo de investigación en tanto que es una comunidad aprendiente, donde interactúan docentes y discentes en la construcción del conocimiento funcional, significativo y pertinente con el cual es posible afrontar los desafíos profesionales.

El objeto de este estudio se centró en conocer y comprender las competencias que el colectivo docente pone en juego en su quehacer didáctico, tanto las explícitas como aquellas menos visibles pero que dejan su impronta en la toma de decisiones prácticas y que subyacen entre el colectivo profesional.

Tratando de sobrepasar las modas al uso respecto de las competencias, se pretendió profundizar en la comprensión de aquellos significados que subyacen en los discursos y en la toma de decisiones en los contextos y procesos de enseñanza-aprendizaje promovidos en la UNA.

El trabajo de campo se inició en el último semestre del 2010 y a lo largo de todo el periodo lectivo del año 2011 momento en el cual culminó. Con el fin de cumplir con los objetivos planteados en esta investigación y de acuerdo con la metodología seleccionada (cualitativa), se recurrió al uso de distintas técnicas, tales como: a) entrevistas semi-estructuradas, b) observación participante, c) grupo focal, d) cuestionario y e) análisis de documentos, para luego cumplir con la triangulación de la información recopilada por esos medios; entonces, la reflexión y la crítica se constituyeron en elementos claves para la interpretación y el contraste de los insumos obtenidos en el seno mismo del proceso de investigación y del accionar con el grupo.

Cabe mencionar que los participantes en cada una de las técnicas se seleccionaron mediante un muestreo de tipo intencional. La muestra o subconjunto de la población de docentes y de estudiantes seleccionada se obtuvo en los ocho centros de estudio (en la UNA reciben el nombre de Unidades Académicas y la Facultad de Ciencias Sociales tiene además de estas dos centros de investigación) producto de los datos facilitados por el personal de gestión.

El criterio para la selección de la muestra, la cual fue de un total de 36 participantes, se relacionó con los datos obtenidos en cada unidad académica previos a la realización del trabajo de campo, momento en el cual se procuró establecer relaciones entre el discurso promovido por los protagonistas del proceso educativo (docentes y discentes), las actitudes tomadas ante la relevancia del aprendizaje construido y la incidencia que ambos tienen con las competencias (capacidades personales, sociales y profesionales) puestas en juego en el escenario educativo (Gimeno, 2008; Zabalza, 2007; Tedesco, 1999; Perrenoud 2005).

\section{Desarrollo}

El análisis del proceso de cambio que afecta a la educación superior en el caso específico de la Facultad de Ciencias Sociales de la Universidad Nacional y su vinculación con el desarrollo de la pedagogía por competencias, así como la aportación desde la literatura científica especializada, 
posibilitaron aproximarnos al conocimiento del enfoque educativo por competencias, detectar problemas y limitaciones, construir relaciones y elaborar conceptos para hacer una aproximación de las cualidades de la docencia universitaria y la formación pedagógica a partir de la propia experiencia e ideas de los protagonistas en el proceso. Se hace necesario recordar que dichos protagonistas están llamados a contribuir en el desarrollo de destrezas y formación que garanticen la inclusión del estudiantado en los principales núcleos de la gestión política, económica y social del contexto, resultando de ello una mayor posibilidad de progreso de la calidad de vida. Estas consideraciones son valoradas como demandas puntuales que la sociedad de la información y del conocimiento le hacen al contexto universitario, de allí la importancia de que sean analizados los procesos educativos a la luz de un mejor empleo de los recursos académicos para la formación de las nuevas generaciones.

Por la escasez de antecedentes teóricoconceptuales sobre el objeto investigado (las competencias explícitas y subyacentes promovidas en las prácticas docentes) dentro de la UNA y específicamente en la FCS, así como en el resto del país costarricense, se procedió a realizar una extensa reflexión y análisis en forma de estudio de caso acerca del papel que desempeña en la educación superior uno de los temas más controvertidos a los que se enfrenta la universidad: el desarrollo de competencias en los procesos educativos como las vías requeridas para enfrentar de manera adecuada los límites y las posibilidades de la institución universitaria para promover aprendizajes útiles, relevantes y pertinentes como elementos claves para el desarrollo en la sociedad del conocimiento.

El marco de referencia del problema investigado fue que la educación actual se diferencia de la tradicional en la medida en que la práctica vinculada con la teoría se hilvana para construir conocimiento con significancia, incumbencia y aplicabilidad. La función de la acción pedagógica supone centrarse en garantizar al estudiantado y a la sociedad una formación amplia, integral, profunda y relevante, vinculada con las necesidades del entorno y dimensionada desde los fines de la educación en la práctica. Lo que hace que se consideren los procesos de enseñanza y aprendizaje como los responsables de dirigir la práctica pedagógica para esa construcción diferenciada del conocimiento. Para ello y parafraseando a Pérez en Gimeno, Pérez, Martínez, Torres, Angulo y Álvarez (2008), se cree, deben cambiar las formas de entender el conocimiento, así como la formación personal, social y profesional de aquelos implicados en el acto educativo.

Desde esta perspectiva y según el discurso contradictorio de las competencias como modelo de enseñanza aprendizaje que nos exponen diversos autores (Gimeno, Pérez, Martínez, Torres, Angulo y Álvarez, 2008; Barnett, 1994; Perrenoud, 2005; entre otros), entendemos que para algunos este marco de referencia es concebido como parte de los indicadores del desempeño para su concreción, puesto que la competencia es valorada como una cualidad que se tiene, se adquiere o se es capaz de mostrar o demostrar, a partir del desarrollo de destrezas técnicas que le permiten al individuo operar o ejecutar una acción determinada en un momento concreto. Esto nos recuerda el modelo de formación por objetivos, lo que se podría traducir en un paso atrás en el proceso de planificación de las prácticas promovidas en los contextos de enseñanza.

Otros por el contrario relacionan el modelo en cuestión con el desarrollo gradual de habilidades cooperativas y de socialización en el aprendizaje, la cual se alcanza como consecuencia de la capacidad holística del ser humano para integrar sus atributos personales (cognitivos y metacognitivos) y así dar respuesta a las demandas del contexto (profesionales, personales y sociales). Es valorada como un proceso que no concluye, en donde cualquier competencia es mejorable a lo largo de la vida y según los condicionantes del contexto que se presenten.

A la luz de lo expuesto en esta última valoración sobre las competencias como proceso de construcción permanente, mejorable y de integración holística de los recursos cognitivos y metacognitivos del ser humano, se entiende que las instituciones de educación superior deberían tomar algunas consideraciones como se expone a continuación: 
1) Abordar las competencias como marco de referencia para seleccionar contenidos, transformar la evaluación y los procesos de enseñanza aprendizaje, esto conducirá a tomar en cuenta el enfoque constructor de una colectividad, capaz de nutrirse de forma autónoma de las realidades provistas por el entorno, de los saberes que su experiencia de vida le han dado, de la teoría y convivencias promovidas en los contextos de aprendizaje formal, todas ellas responsables de contribuir a la búsqueda de soluciones para resolver las situacionesproblemas que emergen del entorno.

2) Conceder significancia y funcionalidad a los aprendizajes conduce a una forma diferente de entender la evaluación promovida en los contextos educativos, estableciéndose en un elemento crucial para que el educar por competencias se constituya en la vía para el entendimiento de aprendizajes relevantes. A su vez, le permitirá al estudiantado conocer nuevas formas para empoderarse del conocimiento con el cual podrá influir de manera proactiva y permanente en su vida personal y profesional.

3) Valorar la pedagogía de las competencias como un enfoque promotor de aprendizajes para la vida, podría contribuir a contrarrestar el efecto resultante de las opiniones encontradas y las contradicciones significativas que ha despertado entre los artífices del proceso educativo, con lo cual aun habiendo calado en el discurso de los teóricos, pareciera no impactar del mismo modo la enseñanza y la práctica docente aún por la polémica que provocan algunos conceptos.

Asimismo y tomando como referencia los datos emergidos del estudio realizado en el escenario universitario de la Facultad de Ciencias Sociales de la Universidad Nacional de Costa Rica, se destaca que, de acuerdo con el discurso promovido por algunos docentes y discentes respecto a formar bajo un enfoque por competencias, éstos la consideran más que una transformación profunda, hasta ahora, una moda que ha surgido y pronto podría estar obsoleta. Algunos otros al parecer sí le dan crédito como modelo de enseñanza pero confiriéndole bondades no exentas de ciertos tecnicismos; posición poco congruente con una construcción del conocimiento que supone derivarse del desarrollo integral y autónomo del individuo.

Igualmente significativo fue el sentido que adquiere el aprendizaje, el cual no parece convencer a ninguno de los colectivos implicados. Por un lado, el profesorado afirmó que los estudiantes, en la mayoría de los casos, están carentes de interés y sólo buscan pasar las asignaturas de sus cursos y, por otro, los estudiantes no se sienten satisfechos con las enseñanzas y sus prácticas entre las que destacan el tipo de mediación pedagógica, los contenidos tratados y su utilidad o los espacios facilitados para promover los aprendizajes a la luz de las demandas del entorno que reciben.

De la misma forma, tampoco parece haber quedado claro de la documentación institucional y de su análisis la relevancia de los aprendizajes que caracterizan la formación promovida, siendo este es el eje fundamental sobre el que se asienta el discurso de competencias.

Como consecuencia de lo expuesto y con el convencimiento de que la provocación de aprendizajes relevantes y útiles es el motor clave que debe impulsar el desarrollo de competencias, se emprendió un análisis de contenido de los discursos que elaboran los docentes sobre lo esencial de su función y lo que en la realidad de la práctica sucede, para poder dar a evidenciar la relación que subyace entre el pensamiento construido por los dirigentes del proceso educativo y lo que transcurre en el escenario educativo. Para ello, fue también necesario conocer la incidencia de las variables organizativas en la puesta en escena de las competencias, de lo cual se mostraron múltiples razones, como saturación en los grupos, limitaciones para la escogencia del profesorado por parte del alumnado, un modelo curricular expuesto en la documentación oficial que difiere del encontrado en las acciones prácticas, poco reconocimiento a la labor profesional promovida, entre otros, para creer que la gestión universitaria no siempre se desarrolla según las necesidades profesionales y pedagógicas que demanda el entorno, tanto para el beneficio del colectivo docente como del discente. 
El análisis y la reflexión crítica como un ejercicio que contribuye al enriquecimiento de la enseñanza universitaria permitió conocer algunas inconsistencias en el decir y en el hacer docente que podrían estarle impidiendo indagar sobre las formas más asertivas para abordar la enseñanza, limitándole la promoción de aprendizajes comprensivos, relevantes y funcionales, así como también, obstaculizándole la puesta en marcha de aprendizajes conducidos por la investigación y alejando al docente de su función como mediador activo en el proceso de construcción autónoma del conocimiento.

Los datos emergidos del estudio de campo fueron aglutinados en categorías y subcategorías las cuales permitieron analizar y socializar la basta información encontrada:

1. Las competencias y "la novedad" en la enseñanza

\subsection{La innovación en la Facultad de Ciencias Sociales}

1.2. Una concepción tecnicista de las competencias

1.3. La experiencia docente y el cambio en educación

2. Aprendizaje y alumnado

3. Formación y autonomía docente

4. Vocación y desempeño docente

5. Evaluación y competencias

6. Interdisciplinaridad para el desarrollo de aprendizajes relevantes

Como se mencionó, de los anteriores bloques temáticos surgieron múltiples evidencias, algunas de estas sistematizadas de la siguiente manera:

En primer lugar, fue evidente una notoria resistencia al cambio que, en este caso, impulsaban las nuevas orientaciones provenientes del modelo de competencias por parte de un grupo mayoritario del colectivo tanto docente como discente participante en la investigación debido, entre otras cuestiones, a las siguientes causas:

- Fuerte homogenización en el uso de métodos didácticos.
- Inexistencia de una formación inicial bien fundamentada en la importancia de la innovación educativa.

- Enseñanza más centrada en planteamientos teóricos que en procesos de intervención.

- Escasa intención de querer desaprender y poder así reconstruir el proceso de enseñanza aprendizaje.

- Fuerte tendencia a promover aprendizajes desde la disciplinariedad.

- Predisposición por darle al aprendizaje y al desarrollo de competencias, un carácter utilitario lo que impide su despliegue o movilización en otras realidades para contribuir al bienestar social que se busca suscitar a partir de la innovación: "En el trabajo le piden a uno que sepa cómo hacer las cosas, especialmente aquellos procesos que son nuevos, innovadores, pero en la universidad no nos los enseñan, ¿cómo se puede entonces ser competente?, yo digo esto porque mi mamá estuvo aquí estudiando lo mismo que yo y me dice que los cursos y la forma como los dan no han cambiado a pesar de que los contenidos que se ven en clase ya ni se relacionan con lo que pide la empresa".

- Poco deseo por hacer del conocimiento la centralidad del proceso de enseñanza-aprendizaje.

- Débil formación docente para poder revelar con maestría la función principal de su mediación: "la universidad no te enseña a dar clases...".

- Desconocimiento, por parte del alumnado, sobre la funcionalidad y utilidad de los aprendizajes, mostrando una tendencia a conceder a los mismos un valor de cambio.

- Limitada intencionalidad con la que se planea y promueven los aprendizajes, "Yo siempre me he preguntado, hasta donde realmente se está innovando. Cada uno de nosotros hace lo que puede, pero la verdad es que la mayoría cuando están en eso ni valoran el perfil de salida del estudiantado".

- Insuficiente compromiso docente para asumir un papel significativo como mediador activo del proceso de enseñanza aprendizaje. 
A la luz de estos datos, pareciera necesario transformar los intentos que se realizan para abordar la mediación de los procesos de aprendizaje por una en que se permita mejorar sustantivamente la relación entre el valor de la disciplina y la práctica pedagógica. Asimismo, se cree importante tomar en cuenta la globalidad de los aprendizajes para el manejo que el docente haga de los criterios teóricos fundamentales, esto con el propósito de dirigir una formación para la complejidad, para la autonomía del saber y para la intervención cognitiva que la sociedad requiere.

El camino para el análisis de realidades estudiadas a la luz de una síntesis teórica de las competencias básicas, podrá ser moldeado y reconstruido como parte de un proceso gradual para el entendimiento y posterior accionar del colectivo estudiantil. Lo contrario significaría una débil fundamentación teórica la cual podría evitar que se logre el dominio de algunas habilidades previas de interpretación y comprensión de los asuntos objeto que el desarrollo del conocimiento autónomo requiere. Se considera que los aprendizajes promovidos desde una pedagogía por competencias parten de una transformada manera de valorar, entender y hace funcionar el conocimiento que se debe construir autónomamente en el seno del proceso. En resumen, esto es de lo que se trata, y a la vez es lo novedoso por las relaciones que se establecen. La significancia del aprendizaje es resultado de la vinculación que subyace entre teoría y práctica, en una relación dialéctica, sin ser una más importante que la otra, sino complementarias.

De allí que se valore cualquier propuesta de cambio en la educación desde su esencia y relevancia, lejos de convertirse en un simple maquillaje o variación de lo existente, debe fundamentarse con solidez, claridad y bajo la premisa de la funcionalidad, puesto que como se ha evidenciado en este estudio hay quienes consideran la propuesta en cuestión "lo mismo de lo mismo" lo que conduce a posicionarla bajo un simplista esfuerzo empírico y teórico.

En segundo lugar, debido a que lo que distingue el enfoque por competencias de otras propuestas de cambio educativo es el valor concedido a la construcción del conocimiento, será importante valorar lo siguiente:
- Otorgarle un diferenciado trato a la escogencia de los contenidos provenientes de la pluralidad cultural (el saber, las formas de expresarnos y comunicarnos, la ciencia y la tecnología, el arte, entre otros) ya que estos servirán de insumo para promover aprendizajes funcionales y de incumbencia a partir de los cuales se puedan desarrollar estrategias para enfrentar cualquier situación nueva o desconocida que se presente en la vida.

- La pertinencia en el uso de saberes y recursos interrelacionados con habilidades y actitudes, como abono sustancial para mejorar la vida profesional y personal del ser humano, a partir de su desarrollo intelectual, afectivo y moral.

- La profundidad y permanencia con la que acompañan los aprendizajes al ser humano desde una dimensión controvertida, la cual provoca hacer lectura de las incertidumbres del entorno sin dejar de lado el reconocimiento de la humanidad del ser.

- El sentido dado al carácter metadisciplinar de algunos de los componentes procedimentales, actitudinales y conceptuales que involucran los aprendizajes para la construcción de competencias que permitan darle funcionalidad a lo aprendido sin dejar de criticarlo, transformarlo o hasta desecharlo si fuese el caso.

Tomando en cuenta que el desarrollo de capacidades básicas y meta-específicas en el individuo, como la vía facilitadora de la construcción del conocimiento, debería hacerse al compás de las exigencias sociales, lejos de una concepción de recurso unívoco y rígido. Según se evidenció, una de las situaciones que obstaculizan la viabilidad de una formación liderada por el desarrollo de competencias es la imposición de este enfoque sin su debido tratamiento para poder ser parte de las ideas profesionales, personales y propias de la socialización docente, lo cual dicen Hargreaves y Fullan (2012), es lo más difícil de cambiar porque es lo más asentado. Esto podría estar teniendo cierto protagonismo en los discursos sobre la práctica emitidos por el colectivo docente, los cuales se desarrollan sin solidez y concreción, por lo que será ingenuo pensar que el práctico (grupo 
docente) la vincule a su metodología de trabajo como muestra de su empoderamiento.

Amparados en la "moda", algunos educadores justificaron la implementación del enfoque de enseñanza por competencias, pero sin denotar gran afinidad y convencimiento de los aportes de dicho enfoque en la construcción del conocimiento. Por tanto, fue posible percibir que desde una vacía concepción se le percibió como una simple novedad de formación, que no está concretizando ni impactando los contextos de aprendizaje ni el ejercicio de la labor docente.

En tercer lugar, se defiende que son muchos los factores que "se confabulan" para que las competencias se posicionen como un enfoque de enseñanza que guíe la práctica formativa, de allí que se concibiera como fundamental el acompañamiento institucional para la puesta en marcha de innovaciones educativas provocadoras del cambio puesto que generan complejidad de pensamiento como resultado de los conflictos cognitivos que despiertan en el pensamiento del estudiantado como consecuencia de su carácter esencialmente contextual.

Por tanto, se esperaba que las políticas institucionales fueran las líneas generales, el marco de referencia para el accionar docente, las cuales respondieran sustancialmente a las realidades del entorno, transformándose sistemáticamente para contribuir a la puesta en marcha de un currículo flexible, desafiante y actualizado; del cual se suponía debían derivarse prácticas pedagógicas congruentes a las demandas de la sociedad del conocimiento.

En el transcurso de la investigación, fue otro el aporte que reflejó la política institucional. Se pudo valorar la existencia de una necesaria flexibilidad en los agrupamientos estudiantiles (se los cuales evidenciaron una cantidad de grupos que superan los 40 alumnados, especialmente en la carrera de Administración de empresas), así como también, el requerido sometimiento a valoración de la excesiva carga lectiva que tiene el profesorado (la mayoría del personal en propiedad labora 40 horas semanales de las cuales según la normativa de contratación de la UNA debe dedicar entre 12 y 14 horas a la clase presencial, 4 horas a la atención individual del estudiantado, 14 horas a la preparación de lecciones y evaluación, 4 horas a la sistematización y producción didáctica, 4 horas para reuniones y comisiones y 2 horas a la actualización y capacitación), esto hace difícil que el colectivo docente cuadre sus tiempos y pueda involucrarse en iniciativas de formación permanente que beneficien su desarrollo profesional. Ambas consideraciones deberán ser analizadas desde el área organizativa de los centros y unidades universitarias, tomando en cuenta que limitan o reducen el tiempo para la formación.

Al mismo tiempo, se cree que la masificación estudiantil dificulta la participación directa en el aula, puesto que no contribuye a una práctica reflexiva y a una atención más individualizada del alumnado (para ello no todas las unidades académicas cuentan con despachos o cubículos para atender a la población de manera unipersonal), además de la precariedad de algunos de los medios didácticos que son facilitados (las aulas no se encuentran equipadas con tecnología multimedia, se cuentan solo con un televisor en cinco aulas del edificio de la Facultad, algunas unidades académicas cuentan con muy pocas portátiles y retroproyectores que debe ser solicitados con mucha antelación para su préstamo debido a la alta demanda de este equipo).

Igualmente relevante resultó la escasa promoción del trabajo colaborativo desde la institución y entre los profesionales (no hay políticas de trabajo interdisciplinario, los cursos colegiados cada vez son menos, las condiciones laborales de interinazgo hacen que el personal asista solo a dar su curso sin que se denote un interés legítimo y real por trabajar con otros), esto hace que el individualismo y el aislamiento sean características del ejercicio profesional del docente en esta universidad lo cual dificulta la investigación compartida y las actividades de mejora personal e institucional, demandadas para una efectiva cooperación y coordinación en los procesos de intervención y práctica pedagógica.

Por otro lado, para hacer posible un cambio en la enseñanza por competencias la institución debería atender al desarrollo profesional del docente en aras de fortalecer el proceso de aprendizaje promovido, por cuanto se evidenció que existe una estrecha relación entre dicho desarrollo y los niveles de motivación manejados 
por el colectivo docente, condición responsable de incidir en beneficio de su función, "Yo he recibido cursos y realmente son muy buenos, lo actualizan a uno, te permiten hacer conciencia de la verdadera función social que tenemos y no tanto tirado a lo disciplinar, lástima que no todos nos disponemos a llevarlos, sería bueno que fuera una disposición institucional, claro bien consensada no a golpe de tambor como algunas otras cosas que se nos imponen en la Universidad", para otros la realidad es diferente, y quizás no tan positiva respecto a las oportunidades que se le brindan para formarse paralelo al desempeño de su práctica y el reconocimiento que estas tienen n la universidad, “...y para qué.......no sirven para nada" y "Aquí es uno el que mantiene viva la llama... bueno y los estudiantes que te buscan y te motivan, porque la Universidad no te lo reconoce y eso es doloroso". Tal y como vemos, algunas de las percepciones docentes y discentes registradas como parte del trabajo investigativo señalaban la ligereza y falta de importancia que le otorgan los docentes a los procesos de formación y al sentido que éstas tiene en la vocación como construcción de su profesionalización.

Fueron los estudiantes quienes detallaron de forma puntual como es que la universidad deja de lado lo sustancial, o sea, el reconocimiento de una "buena" labor docente, y se centra en muchas ocasiones a atender cuestiones meramente operativas, olvidando que, como institución educativa, debe también ser el modelo que ha de promover el desarrollo socio-cognitivo en aquellos sujetos en formación, a los cuales debe desarrollarles sus potencialidades futuras, sus valores y su calidad ética como seres humanos.

Entonces, se podría pesar que entre los llamados que la sociedad costarricense le hace a la gestión universitaria contemporánea estaría el centrarse en la idea de que ésta debe esforzarse permanentemente por:

- Mejorar los procesos administrativos y gerenciales que conduce.

- Elevar su calidad de servicio nucleando los saberes con mayor incumbencia y funcionalidad.

- Satisfacer las necesidades y expectativas de la población que atiende.
- Buscar y hacer respetar su autonomía.

- Comprometerse con la liberación o descolonización mental del pueblo.

- Proveer una atmósfera en donde el valor concedido al desarrollo humano sea altamente reconocido y apreciado.

- Emplear mejor sus recursos académicos para una mayor asertividad en la formación de las nuevas generaciones para lo cual se requerirá distanciarse de modelos educativos tradicionales en donde lo que predomina es la memorización y transmisión de contenidos y no la construcción integral del ser humano; esta última como orientación que destaca al modelo por competencias.

En cuarto lugar, las responsabilidades que debe atender la función docente son además de complejas pocas veces reconocidas por algunos de los artífices del proceso y la institución educativa para la que labora. Según se evidenció en el estudio en cuestión, algunos docentes siguen creyendo en la importancia de ser ellos quienes manejen con exclusividad el conocimiento, entendido y asociado casi en la totalidad de las opiniones emitidas al estudio disciplinar. Igualmente relevante sigue siendo (desde la tradicional forma de percibir las prácticas pedagógicas) para el grupo de profesionales participantes el concebir al grupo estudiantil como receptor del aprendizaje, limitado a escuchar y recrearse de las complejas demostraciones de sabiduría del maestro, como esencia misma de la función docente, "yo doy mi clase y el que quiere aprender que aprenda", asimismo otros, se declaran conocedores absolutos de las necesidades y actitudes de todas y cada una de las poblaciones estudiantiles a su cargo afirmando, "ya son muchos años y siempre es lo mismo, no quieren aprender" .

Lo anterior reveló la existencia de una conducta docente que se disocia del sentido primordial de promover aprendizajes que tomen como referencia el desarrollo de competencias básicas, puesto que impide el protagonismo del estudiantado en el proceso, limitándole sus actuaciones y obviando la importancia de un procesamiento de la información significativa y funcional, de esa manera, se cree, podría estarse desplegando un velo que no deja conocer y 
orientar aprendizajes relevantes que conlleven a facilitar las herramientas necesarias para el desarrollo personal, social y profesional acorde con las demandas del presente.

Tal y como se pudo observar, a partir de construcciones como las expuestas, se ha ido gestando entre el profesorado creencias que alimentan su discurso práctico y a las cuales hay que prestarle la debida atención, ya que podrían estarse transformando en obstáculos que impiden el paso al interés por aprender, por indagar y por mantener siempre viva la curiosidad en el alumnado, quien también con su opinión indica no sólo malestar, sino desinterés, ante la falta de significancia que el proceso le merece.

El sentido del enfoque por competencias es fortalecer por medio del incesante deseo de construir conocimiento para dar respuesta a los conflictos y problemas que afloran en la cotidianidad de la vida. Bajo esta orientación pedagógica, los aprendizajes se edifican a la luz de constructos teóricos que cobran una renovada vida a partir de las dudas, de las realidades y de las necesidades que surgen en los contextos de aprendizaje formal e informal. Como efecto, es de suponer que se genere empoderamiento y consecuentemente autonomía del conocimiento por parte del estudiantado. Por eso el creer en la necesidad de que el estudiante se sienta pleno en su entendimiento para poder contribuir al proceso de enseñanza aprendizaje en la doble vía, en donde se le facilita y promueve la construcción y reconstrucción del conocimiento, a la vez, que se le alimenta su deseo de aprender a aprender.

En el caso estudiado, la poca claridad con la que se ejemplificaron los contenidos tratados en los contextos de aprendizaje podría ser el resultado de un modelo formativo de débiles prácticas intelectuales, de investigación e implementación, las cuales contribuyen a vetar la construcción del conocimiento en lugar de promoverlo y fundamentarlo en aras de evidenciar su relevancia y utilidad. Lo anterior lleva a pensar que es necesario formar un colectivo docente con capacidad de conciencia sobre la importancia de otorgarle al conocimiento y al discente la centralidad requerida en el proceso. Lo fundamental será planear el deber-hacer didáctico a partir de una consensuada vinculación entre teoría y práctica, en donde se haga reverencia a los distintos significantes de identidad (distintivos fundamentales en la sociedad del conocimiento) como elementos claves para la contextualización, pertinencia y significancia requerida por los estudiantes para construir un pensamiento propio como respuesta a las demandas de la sociedad del presente, esto por cuanto se parte del hecho de que lo aprendido debe ser empoderado para el logro del desarrollo autónomo desde una práctica que construye y reconstruye teoría.

Así será posible dar respuesta a la exigencia de que el estudiantado contraste realidades a la luz de una orientación conceptual base, transformable y edificada para la significancia y funcionalidad. Esto es un llamado hecho desde discursos discentes que indican "con sólo que pida las páginas vistas es suficiente", en donde se evidencia la importancia otorgada a la transmisibilidad del conocimiento, orientada por la memorización y la escasa autonomía constructiva del conocimiento.

La tradicional forma de pensar y hacer del acto educativo está en la transmisión y la memorización de teorías prestadas, realidades que hoy son objeto de crítica y causantes de la deslegitimación de la función docente. Su despliegue en los contextos aprendientes podría no ser cosa del ayer, por el contrario, al parecer todavía se reconoce su protagonismo según el tipo de prácticas pedagógicas promovidas. Muchas de las observaciones realizadas como parte del trabajo de campo requerido para este estudio, pusieron en evidencia la reiterada insistencia de hacer del contexto de aprendizaje un espacio para demostraciones por escrito o en el mejor de los casos orales, en un tiempo limitado y bajo contenidos impuestos por el conocimiento que el profesorado tiene sobre diversos conceptos enlatados, los cuales en la mayoría de los casos se presentaron tal y como quien les dio origen las concibió.

La contextualización, la significancia, la funcionalidad y la relevancia de los contenidos tratados en los entornos aprendientes, parecieron no dar tregua a la construcción y reconstrucción del conocimiento en colectividad, “...nos cuesta aterrizar en la práctica ya que aunque en un futuro se supone que vamos a ser profesores de esa disciplina, lo que en los cursos de pedagogía 
nos han enseñado son puras teorías, las cuales aunque son importantes no nos aterrizan en el aula, donde vamos a trabajar como profesores...". Asimismo, la esencia problematizadora de los aprendizajes promovidos en una formación por competencias no llega a constituirse aún en la realidad pedagógica analizada. Por el contrario, lo observado en las dinámicas metodológicas de clase, así como los temas desarrollados, no dejaron de revelar prácticas más allá de las tradicionalistas (predominio de la transmisibilidad, la memorización y la uni-direccionalidad, entre otros), "Los profes tal vez sepan mucho, pero a la hora que explican cuesta entenderles".

Lo mencionado es un llamado a la universidad, como la institución que urge de construcciones propias del saber, las cuales suponen desarrollarse como producto de aprendizajes globales derivados del contraste de realidades y saberes, así como también, es un aviso a la función docente, la cual tiene un componente moral, el cual deberá ser valorado como elemento esencial de su condición profesional.

En quinto lugar, otro de los aspectos que evocaron confusión en la iniciativa de cambio propuesta era el sentido concedido a la evaluación. Pensemos que en todo este proceso de cambio la evaluación juega un papel fundamental modelando y direccionando la dinámica educativa, tal y como afirma Pérez, Soto, Sola, y Serván (2009),

Los modos de evaluar los aprendizajes de los estudiantes condicionan sustancialmente los procesos de enseñanza de los docentes, la selección de los contenidos del currículo, la determinación de las prácticas de enseñanza y sobre todo la configuración de las experiencias y estilos de aprendizaje de los estudiantes, así como el clima de relaciones sociales y los ambientes de aprendizaje escolar. (p. 93-94)

En consecuencia, es posible creer que los centros de formación son los llamados a transformar su tradicional forma de concebir la enseñanza y pasar de una que es asignaturizada, centrada en contenidos arbitrariamente seleccionados (muchas veces producto de preconceptos sobre los supuestos saberes por trasmitir en los salones de clase para que el alumnado este "bien preparado" para el trabajo), en donde se deprecia el valor de la práctica sobre la teoría y se centra en el desarrollo del saber por el saber, a una en donde lo protagónico sea el desarrollo de capacidades que le permitan al sujeto transferir y aplicar los conocimientos desarrollados en múltiples situaciones-problema que el proceso de evaluación, elemento clave de formación, le presenta.

El profesorado, en su mayoría, así como la documentación institucional, reflejan estas características de la evaluación: flexible; gradual; dinámica; compleja; permanente; emancipadora del saber; facilitadora de la autonomía del pensamiento y del hacer; reveladora de la estrecha vinculación entre práctica y teoría y constructora de conocimiento a la luz de respuestas impredecibles pero sólidamente argumentadas.

A pesar de las características anteriormente señaladas utilizadas en el modelaje de la evaluación y los beneficios que estas suponen al desarrollo de la práctica del aprendizaje como proceso emancipador del saber hacer y ser, se tomó en cuenta las limitaciones evaluativas y la complejidad que caracteriza cualquier proceso de transformación de los procesos de enseñanzaaprendizaje en donde la evaluación cuenta con un protagonismo indiscutible.

Lo sustancial en cualquier transformación de los entornos aprendientes, depende entre muchas otras cosas de las decisiones evaluativas tomadas a la luz del desarrollo curricular (políticas y prácticas). Estas suponen incidir en la transformación de la actitud docente por una más reflexiva, analítica y propositiva respecto a su práctica. No obstante, podría ser dificultoso que encuentre las vías alternas para trasladar dichos cambios al modo como le condiciona el proceso de evaluación, situación que es resaltada por el estudiantado: "A uno le enseñan de una forma y el examen se lo hacen de otra". A lo largo del trabajo investigativo fue posible notar que en muchas ocasiones, aunque se intentan introducir algunos cambios en la metodología o en la selección de contenidos, la evaluación sigue siendo considerada como un elemento sancionador en contra del alumnado: "Aunque uno sepa, el resultado del examen es el que manda, esa siempre ha sido la forma de evaluar", obstaculizando la puesta en marcha de iniciativas para el cambio en profundidad. 
Aparentemente, siguen sopesando las rigideces curriculares en la práctica pedagógica y a su vez obstaculizando acciones de formación mucho más enriquecedoras, orientadas a desarrollar en el alumnado conocimientos duraderos que nutran su vida de forma integral, procurando en todo momento la promoción de aprendizajes relevantes y funcionales.

Todo proceso formativo supone contar con una base curricular suficientemente flexible y actualizada, promotora de un estilo de enseñanza-aprendizaje que dote al estudiantado de herramientas para responder a los problemas emergentes en su vida personal y profesional, hecho que redundará en la forma de entender y practicar la evaluación.

Las acciones de enseñanza, aprendizaje y evaluación (ambas valoradas de relación dialéctica) no deben concebirse de forma aislada, por el contrario, suponen incorporar conocimientos previos del colectivo en formación, de tal forma que cuando se vea expuesto a nuevos asuntos logre conectarlos a los conceptos preexistentes para poder utilizarlos como propios, con bastante claridad, pertinencia, significancia y disponibilidad, así lo destaca el colectivo docente cuando afirma: "Yo creo que es necesario que dejemos de estar creyendo que el estudiante llega ahí sin saber nada, al contrario, debemos de valorar y partir de todo el conocimiento que la vida y la escuela le ha dado, hay que respetarlos son personas que les ha costado llegar hasta nuestras aulas y tratarlos como ignorantes es para mí un irrespeto total".

Aunque existen docentes dispuestos a valorar la evaluación como parte esencial del proceso formativo, existen algunos otros que dicen sentirse maniatados por la inflexibilidad del sistema en cuanto a la evaluación se refiere, se cree que lo más significativo de esto es su rigidez de actitud, la cual le impide explorar nuevos formas de evaluación lejanas de una mera calificación del alumnado, "Uno tiene la responsabilidad de hacer la prueba y ellos de estudiar para contestarla". Afirmaciones como esta se aleja de las intenciones legítimas de hacer de la evaluación un componente fundamental y desafiante del proceso educativo, por el contrario, podría más bien constituirse en freno de la función formativa.
No es justificable que ante un mundo que clama por aprendizajes sustanciales y aprovechables para la sociedad, se siga llevando a cabo el discurso de que "la flexibilidad es relativa amparado en esta poca dinámica visión curricular. El colectivo docente insiste en que no hay nada más que emprender una acción formativa en donde "El que sabe, sabe", haciendo del examen la meca de la evaluación, "El examen lo dice todo", poniendo de manifiesto una confusa responsabilidad por parte del profesorado ante los aprendizajes que promueve y su vinculación con la construcción del conocimiento como mediador activo del proceso.

Lo anterior nos conduce a pensar en la posibilidad de que el docente siente que una cosa es su pensamiento ético y otro dar clases en la universidad, así como también, que siga considerando el conocimiento como algo abstracto y ajeno al sentir cotidiano. Estas situaciones podrían ser parte de la aparente dificultad que tiene el combatir la inercia y la sumisión en el ejercicio y cumplimiento de la función educativa.

Se cree entonces que la universidad como institución social y el profesorado como su representante en la práctica pedagógica deberán velar porque la evaluación se constituya en un recurso formador para el desarrollo y bienestar de las personas y su entorno. Como consecuencia, debería estarse conduciendo al colectivo docente a plantear un sistema de evaluación con una orientación visiblemente formativa y continua, capaz de transformar los criterios de selección de los contenidos en aprendizajes relevantes a promover en los contextos universitarios y así poder desarrollar competencias en el alumnado, en donde la comprensión, la interpretación y el análisis, en vez de la memorización y repetición, sean los criterios a tomar en cuenta.

Para identificar las herramientas para evaluar el proceso como un todo, sin fragmentarlo, con miras a construir saberes, no a medir (por lo difícil y poco efectivo que es) o a etiquetar al estudiantado, podría constituirse en uno más de los retos de la función docente, ya que supone identificar las vías para integrar la evaluación al proceso cotidiano de enseñanza-aprendizaje, estructurando un sistema de características útiles y funcionales para el mejoramiento del proceso 
de formación, el cual deberá estar al servicio del aprendizaje y del perfeccionamiento profesional del profesorado.

En sexto lugar, la formación del colectivo docente es hoy más que nunca valorada como la llave a la reflexión de la práctica y a la búsqueda de ingeniosas formas de centrar la construcción del conocimiento.

La naturaleza de la formación del docente debe tener características permanentes, las cuales se identifican como el motor que transforma el entendimiento y la implicación del profesorado en su labor educativa como el medio de influencia en el desarrollo de la sociedad.

La transformación del pensamiento y del actuar del profesorado deberán ser las banderas que se agiten como consecuencia de su formación permanente. El pensar y el hacer del formador deben entenderse a partir del manejo discursivo que este haga en su práctica de clase. El diálogo promovido por el grupo profesional en torno a su acción pedagógica será instrumento fundamental que incidirá en el desarrollo vocacional de su profesión, a manera de respuesta a las demandas impuestas por la sociedad como docente universitario.

Según fue evidente a lo largo del estudio realizado, se denotó que se da un débil impacto de los procesos de formación docente para el mejoramiento de la práctica promovida. Algunos profesores participantes manifestaron menosprecio por dichos procesos, "para mí no sirven de nada", así como también, un sentido conformista, "para que no digan que uno es un anticuado", igualmente, una desorientada intención "lo que importa es que yo sepa", otros aluden a la falta de reconocimiento de sus aportes en su labor profesional "al final nadie reconoce", todo esto pareciera estar generando desidia en dicho colectivo y esta a su vez podría estar creando una mampara que le facilita el perder de vista lo sustancial de su ejercicio en la construcción del conocimiento.

Tal y como se hizo evidente existen ciertas manifestaciones que son manejadas como realidades en la práctica pedagógica, impactando indudablemente el accionar docente. Dichas creencias direccionan la práctica formativa lejos de una educación -centrada en el estudiantadocomo sujeto primordial del proceso de enseñanza aprendizaje. Algunas de estas formas discursivas del pensar y hacer del profesorado están impregnando los contextos aprendientes.

Si bien es cierto, que el sistema educativo pareciera presentar una desarticulación entre las políticas que supuestamente lo sustentan y los mecanismos efectivos para su implementación, esta situación podría deberse fundamentalmente a la falta de continuidad otorgada a los procesos de formación, al escaso interés del profesorado por su crecimiento profesional (quizás por la falta de estímulo institucional y reconocimiento social), falta de tiempo para formarse, insuficientes recursos económicos destinados a los programas de formación, la carencia de convencimiento para transformar la actitud ante la relevancia de aprender a aprender, aprender a hacer, aprender a conocer y a aprender a ser, así como la forma poco intencional (con esto no estamos diciendo que no deba ser consensuada, por el contrario, el profesorado debería estar a gusto con la decisión debido a que hay un claro entendimiento de los beneficios que eso le significa para el mejoramiento de sus prácticas) y planificada de los temas a bordar en los procesos así como también los procedimientos desplegados para su aprovechamiento: "Es doloroso saber que se invierte tiempo y hasta disposición en capacitaciones que no tiene ni pies ni cabeza, uno no entiende para que sirve lo que te dan".

A pesar que se está en un mundo cambiante de gran impacto en la misión formadora de las universidades, la corriente de cambio, para hacer frente a los retos del siglo XXI, pareciera chocar con el muro de la autonomía. Al parecer las transformaciones en esta universidad se han caracterizado por su lentitud y poca congruencia con las realidades del entorno. Es vital que se induzca al consenso a todos los artífices del proceso, especialmente, en un momento cuando las presiones externas (política, económica, social, entre otras) intervienen en la toma de decisiones educativas. Es ineludible una comprensión clara del ámbito de la calidad de la educación universitaria por promover, las competencias profesionales del colectivo docente para su consecución y el papel del discente como pieza fundamental del proceso.

Se defiende que los procesos de formación a los que debería adscribirse el colectivo 
educador son aquellos que le conducirían a estados de análisis y discusión como parte del camino que deberá emprender para autoeducarse. Esto podría contribuir al desarrollo de capacidades que le permitan hacer una mejor escogencia de las competencias y los aprendizajes relevantes que requiere el estudiantado para construir conocimiento autónomo con el cual logre vincular teoría, práctica y realidades del entorno.

Los procesos que reeducan al profesorado deberían nutrirlo de saberes para funcionar efectivamente en su vida profesional y personal, ayudándole a no caer con facilidad en reproducciones fragmentarias y descontextualizadas de su función, permitiéndole ser mediador activo en un modelo de enseñanza que supone ser ante todo integrador, significativo, funcional, liberador y gratificante.

Por ello, el hablar de competencias, en la línea que venimos defendiendo, conduce a desechar cualquier intención de formación bajo un manual o listado de aspectos por hacer, ya que el desarrollo de capacidades requiere práctica, orientación, convencimiento, implicación y sobre todo, transformación de la actitud. Si no atendemos la formación del profesorado con responsabilidad y conciencia educadora para la vida, se estarían alimentando frases tales como "y para qué... no sirven para nada", las cuales desmerecen su importancia para la construcción de conocimiento vivo, de significancia y funcionalidad. De allí que los planes de formación a los que hace referencia el colectivo docente participante, "los cursos son muy buenos, te enseñan cómo quieren que des las clases", parecieran no garantizar el conocimiento de los principios, intenciones y potencialidades del enfoque por competencias, ni mucho menos se constituyen en causa significativa del aprendizaje.

Se cree que el modelo por competencias recurre a estas como recursos entre sí, establecer su diferencia es confuso y fragmentario, así como explicar su vinculación o complementariedad, por lo que formar desde una postura fragmentaria no es coherente con el discurso innovador y transformador de este enfoque, el cual pretende contribuir a la construcción de la autonomía profesional y así no formar parte de discursos tales como "A mí por eso me gusta ir a los cursos para ver cómo es que quieren la cosa, para no nadar contracorriente, ni andar perdido" .

La formación docente y su vinculación en la construcción autónoma del conocimiento para un accionar pedagógico innovador es clave en el desarrollo de las competencias como enfoque de enseñanza aprendizaje.

En sétimo lugar, es importante destacar los cambios que caracterizan a la sociedad contemporánea, los cuales parecieran no haber sido protagonistas en las políticas y prácticas educativas de años anteriores, aun cuando fueron los responsables de determinar las necesidades de formación y el valor que supone tener el aprendizaje en la universidad, motor del desarrollo en los diferentes escenarios del entorno.

La educación como constructora del conocimiento, desde el posicionamiento teórico de algunos autores (Brunner, 2003; Carr, 1996; Durkheim, 2002; España, 2006; entre otros) se concibe como el fundamento de la preparación del individuo para que defienda sus derechos y los de los otros. Entre sus intenciones primordiales están: proponer alternativas de mejora para enfrentar los embates que le impone la mundialización, adoptar un papel protagónico en las decisiones de la ciudadanía en formación, construir conocimiento a partir de las experiencias del cómo entender y hacer en la vida, personal y profesional.

En consecuencia, será necesario que todas las instancias sociales, en especial, la universitaria, unan esfuerzos mediante la puesta en marcha de un acuerdo de orden dinámico, significante, planificado y con representación masiva, en donde se establezca el ideal o el modelo educativo por construir, valorado como la semilla de la cual podría germinar formación para la intelectualidad y, consecuentemente, para el bienestar de la colectividad.

La concepción de educación ha sido evidente a lo largo del presente análisis, junto a la complejidad que supone formar en la actualidad, por lo que al profesorado se le imponen tareas que sobrepasan su conocimiento y entendimiento disciplinar.

Según se pudo denotar en los aportes de expertos en la materia, existe una evidente necesidad de dirigir las prácticas formativas al 
desarrollo del área socio-afectiva del individuo (formar para la sensibilidad), la cognitiva (formar para la intelectualidad) y la metacognitiva (se refiere al cómo aprendemos, pensamos, recordamos y demás), todas ellas preponderantes para un desarrollo integral y sostenible del individuo, sin embargo, quizás lo más complejo de dicha función es la necesidad de empezar por reeducarse como profesional. El docente está llamado a emprender procesos de autoaprendizaje, a fin de ser agente que contribuya a la formación del estudiantado.

Los aprendizajes que se centraban en contenidos específicos, sin ninguna relación con las situaciones o problemas que le aquejan al sujeto, se alejan de la relevancia impuesta por la sociedad contemporánea. Por tanto, el profesorado podría más bien atender a: dominar los contenidos de las unidades de aprendizaje que conforman las asignaturas impartidas, generar un ambiente de aprendizaje acompañado de elementos favorecedores para la atribución de significado y motivar la disposición del alumnado para el aprendizaje.

En consecuencia, el colectivo profesional supone requerir de un cambio en el estilo para ejecutar su trabajo educativo, particularmente, en cuanto a las funciones y responsabilidades que posee para aprender a enseñar y enseñar a aprender, sin olvidar que es la actividad del alumno la que produce los cambios.

En este sentido, es posible creer que la labor educativa en la universidad podría considerar algunos elementos básicos para el desarrollo del proceso de enseñanza-aprendizaje, entre ellos: la generación de espacios para que el estudiantado pueda conocer y cuestionar el origen, sentido y valor de los aprendizajes que se le promueven, a partir de diferenciadas formas de enseñar para no caer en prácticas insignificantes, descontextualizadas, superficiales, y demás: “....al final como que de curso en curso es lo mismo dicho en otras palabras, ...para saber realmente lo que uno tiene que dominar ...metiéndose en internet, ahí está lo que dicen los profes..." ; la estructura de los conocimientos que conforman el plan y programas de estudio (su diseño y despliegue) en donde el alumno es considerado el centro de interés del proceso, en el cual funge como agente activo y responsable de su aprendizaje; y al desarrollo de competencias docentes para el despliegue de prácticas que le permitan al estudiantado movilizar sus recursos cognitivos y metacognitivos (piensa acerca de la resolución de problemas mientras los está a su vez resolviendo) esto con el propósito de que sea capaz intervenir de forma continua en las situaciones problema que surgen del entorno para el mejoramiento de su vida en todos sus escenarios (personal, social y laboral).

En octavo lugar, se valora la interdisciplinaridad como un elemento relevante del modelo educativo por competencias el cual permite que los saberes confluyan y se nucleen para hacer sinergia entre ellos y así lograr entendimiento, "Lo más complicado es lograr que tanto uno como los estudiantes seamos conscientes que para aprender hay que relacionar, mezclar... es decir aprovechar todos los conocimientos a tu alcance, usar todo lo que se pueda...". Este proceso de relacionar los saberes para llegar al entendimiento que se persigue conduce a una acción problematizadora del conocimiento la cual contribuye significativamente en el desarrollo de la capacidad de identificación, producción, transformación, difusión y aplicación alcanzada por el sujeto, como aparte de las tareas que supone llevar a cabo cuando aprende de forma significativa, pertinente y funcional para responder en la vida; así como también, precisa de un transformado abordaje de las prácticas pedagógicas por parte del profesorado quien supone valorar el trabajo en equipo como parte del llamado a la integralidad del conocimiento que la sociedad le hace y el mismo modelo por competencias requiere. El tiempo y la disposición del profesional al desarrollo de las tareas de planeación y el despliegue didáctico ya no se harán de manera aislada, ni persiguiendo proyectos pedagógicos individuales, por el contrario, la práctica será reflejo de las intenciones colectivas que el profesorado tenga respecto a los aprendizajes promovidos. No obstante y según lo reflejado en la investigación, se identificaron algunos puntos débiles respecto al protagonismo de la interdisciplinaridad en los procesos de enseñanza aprendizaje que se promueven en la universidad:

- El escaso o casi nulo trabajo en equipo por parte del cuerpo docente, así como la falta de 
sinergia entre las aprendizajes promovidos en las asignaturas, "No hay relación entre los profes, menos entre las materias".

- La posible descoordinación docente, la cual apunta más que todo, a la disciplinariedad de la acción formadora y no a la interdisciplinaridad. "Yo no sé de ningún profesor que trabaje con otros, cada uno da su clase y ya, es más dudo que todos se conozcan" y "Yo veo que cada uno va por su lado, cada profe ve su curso con ojos diferentes".

- El distanciamiento entre el procesamiento integral de los aprendizajes y lo sucedido en las dinámicas de clase como el trabajo cotidiano, las tareas realizadas y las evaluaciones, "Yo quisiera que las materias tuvieran relación, ya que cuando uno estudia tanta cosa debe como reprogramarse cada vez que entra a un curso, es como si entrara a otra especialidad, como si estudiara un montón de carreras".

Por último y con el afán de seguir avanzando se hace inevitable presentar algunas posibles líneas de investigación que requieren, al menos en la Facultad de Ciencias Sociales de la Universidad Nacional Costa Rica, de mayor y profunda indagación. Las razones que motivan su escogencia resultan de la evidente confusión y falta de abordaje que estas tienen para el mejoramiento de la calidad educativa promovida en los entornos universitarios de esta región centroamericana. Entre las líneas de investigación más destacadas se podrían encontrar:

- El sentido del aprendizaje para el desarrollo de competencias (profesionales, personales y sociales) y su incidencia en la responsabilidad y compromiso social universitario.

- La ética en la formación profesional y en la función del grupo docente.

- Desarrollo profesional, autoconfianza y confianza social del grupo docente.

- La función docente, reconocimiento y selección en los entornos universitarios.

- El impacto de las organizaciones profesionales sobre la identidad y el ejercicio docente.

- La plurifuncionalidad profesional y los procesos de formación docente: enlaces y obtención de experiencias diversas en gestión administrativa, docencia, finanzas, investigación, proyectos y demás fuera del entorno universitario.

- La optimatización de los aprendizajes promovidos en los entornos universitarios: retos, alcances y limitaciones.

- Las competencias docentes para la promoción de aprendizaje autónomo en la educación superior.

- Evaluación formativa para el desarrollo de una pedagogía por competencias.

- La universidad y su función social y económica.

- Sociedad, universidad y democracia: Los retos de un país sin ejército.

- La pertinencia de las ofertas curriculares y su vinculación con la responsabilidad social universitaria.

\section{Conclusión}

El enfoque por competencias como uno más de los modelos educativos universitarios existentes, propone un giro en la forma como se planifica y despliega el proceso de enseñanza aprendizaje. Para este modelo, las competencias son valoradas como un conjunto de recursos (destrezas, conocimientos, habilidades, actitudes, valores éticos, emociones y demás) adecuados al contexto que todo alumnado debe alcanzar para su realización, desarrollo personal, profesional e integración social. Las intenciones perseguidas por este referente educativo se tornan en un desafío más del proceso educativo en la Facultad de Ciencias Sociales, puesto que se esperaba que los resultados del trabajo empírico sobre aprendizajes relevantes dotaran al individuo de herramientas para afrontar y aportar con autonomía a las situaciones suscitadas en las realidades profesionales las cuales demandan ciertas capacidades en el individuo: liderazgo, capacidad de análisis, resolución de conflictos, dinamismo, creatividad, trabajo en equipo, capacidad e interés de aprender a aprender, inteligencia emocional, integridad, pensamiento analítico y conceptual, innovación, entendimiento interpersonal, así como otras. Las anteriores capacidades posibles a ser desarrolladas a partir de este 
enfoque, requieren que el mismo sea valorado como motor de aprendizaje y no como un simple promotor de técnicas que prepara al sujeto para el desempeño de una tarea específica.

Para atender a la trasformación que suponen modelos de mediación pedagógica desarrolladores de capacidades y habilidades para enfrentar la vida con mayores posibilidades de proponer y vencer obstáculos, la universidad misma deberá repensarse para poder convertirse en un centro que forma y se forma a la vez. En donde lo que se hace en el seno de aula es a su vez sujeto de estudio, de diagnóstico y de evaluación continua, pero no aquella versada a cuantificar cuanto aprendió el estudiante, sino más bien, cuanto ha avanzado la universidad (docentes, gestores administrativos, etc) para hacer del contexto un espacio de posibilidades de crecimiento y avance individual y colectivo traducido en mejores prácticas para beneficio de nuestra sociedad.

\section{Referencias bibliográficas}

Barnett, R. (1994). Los límites de la competencia. El conocimiento, la educación superior y la sociedad. España: Gedisa.
Brunner, J.J (2003). Educación e internet: ¿la próxima revolución? Chile: Fondo de Cultura Económica.

Carr, W. (1996). Una teoría para la educación: hacia una investigación educativa crítica. Madrid: Morata.

Durkheim, E. (2002). La educación moral. Madrid: Morata.

España, C. (2006). Educación Superior en Centroamérica. Límites y Posibilidades. Guatemala: Universidad de San Carlos.

Gimeno, J; Pérez, A.I; Martínez, J.B; Torres, J; Angulo y F; Álvarez, J.M. (2008). Educar por competencias, ¿Qué hay de nuevo? Madrid: Morata.

Hargreaves, A y Fullan, M. (2012). Professional Capital: Transforming Teaching in Every

School. USA: Gulf Publishing Company

Pérez, A.I., Soto, E., Sola, M y Serván M.J., (2009). La universidad del aprendizaje:

Orientaciones para el estudiante. Madrid: Akal.

Perrenoud, P. (2005). Diez nuevas competencias para enseñar. Barcelona: GRAÓ.

Tedesco, J. (1999). El nuevo pacto educativo. España: Grupo Anaya.

Zabalza, M.A. (2007). Competencias docentes del profesorado universitario. Madrid: Narcea. 